\title{
Influence of the Break-up on the Fusion and Scattering Processes
}

\author{
J. Lubian, P.R.S. Gomes, R.M. Anjos, I. Padron,'C. Muri, \\ S.B. Moraes, J.J.S. Alves, and A.M.M. Maciel \\ Instituto de Física, Universidade Federal Fluminense, \\ Av. Litorânea, s/n, Niterói, R.J., 24210-340, Brazil \\ R. Liguori Neto, N. Added, \\ Departamento de Física Nuclear, Universidade de São Paulo, \\ Caixa Postal 66318, 05315-970, São Paulo, S.P., Brazil
}

\author{
G.V. Martí, M. Ramírez, A.J. Pacheco, O.A. Capurro, J.O. Fernández Niello, \\ J.E. Testoni, D. Abriola, and M.R. Spinella \\ Laboratorio TANDAR, Departamento de Física, \\ Comisión Nacional de Energía Atómica, Av. del Libertador 8250, 1429, Buenos Aires, Argentina
}

Received on 30 October, 2002

\begin{abstract}
We present results of the study of the influence of the break-up process on the fusion, scattering and reaction cross sections, at near barrier energies. Most of the discussed reactions and scattering mechanisms are induced by the stable weakly bound nuclei ${ }^{6,7} \mathrm{Li}$ and ${ }^{9} \mathrm{Be}$, although comparisons with reactions induced by strongly bound nuclei are also made. We give a picture of the break-up and fusion mechanisms at energies above the Coulomb barrier.
\end{abstract}

The effects of the coupling of elastic, inelastic and transfer channels on the fusion cross section, at energies close to and bellow the Coulomb barrier, have been extensively investigated in the last two decades. At present they are reasonably understood, and their most remarkable effect is the enhancement of the fusion cross section at the subbarrier energy regime, relative to the predictions of onedimensional barrier penetration models. However, in the last years the effect of the break-up process on the fusion cross section started to be investigated, and in the present situation it is still very far from being fully understood. At present there are few and controversial experimental data. From the theoretical side, there are models that predict the fusion cross section enhancement, when compared with the fusion induced by strongly bound nuclei, due to the additional break-up channel. This enhancement should be particularly important at sub-barrier energies, where the coupling effects on the fusion may be strong. On the other hand, some models suggest the hindrance of the complete fusion, due to the loss of incident flux in this channel, caused by the break-up. There are also predictions of fusion cross section enhancement at sub-barrier energies and fusion hindrance above barrier energies, both effects originated from the break-up process.
There is a special interest on this subject due to the recently available radioactive beams of very weakly bound nuclei. Reactions with these nuclei are important in processes of astrophysical interest, as well as in the search of mechanisms that produce super heavy elements. The full understanding of reactions induced by the highly intense beams of stable weakly bound nuclei is a key for the study of the reactions induced by the low intensity radioactive beams. The UFF's group of Heavy Ion Reactions and their collaborators have been investigating this subject during the last years [112].

The most suitable stable nuclei for this type of investigation are ${ }^{9} \mathrm{Be},{ }^{6} \mathrm{Li}$ and ${ }^{7} \mathrm{Li}$, due to their small separation energies that should favor the break-up process. It is important to study the role of the break-up of these projectiles on different target masses, in order to investigate the effect, on the fusion, of nuclear and Coulomb break-ups. Also, it is important to span the energy region from sub-barrier to twice or three times the barrier, because there are evidences that the role of the break-up on the fusion depends on the energy regime.

A major difficulty with many of the fusion experiments is the fact that the complete fusion $(\mathrm{CF})$ and the incomplete fusion (ICF), resulting from the fusion of one of the break-

\footnotetext{
* Permanent address: Center of Applied Studies to Nuclear Development, Havana, Cuba, P.O. Box 6122
} 
up fragments with the target, may not be separated, depending on the experimental detection method used. In this kind of experiments the sum of the cross sections of these two mechanisms somehow masks the effect of the break-up on the complete fusion. Actually, when one studies the breakup process and its influence on the fusion mechanism, at least five different reaction mechanisms should be considered: ( $i$ ) direct break-up that occurs at large distances or large angular momenta. (ii) direct break-up that occurs at short distances or small angular momenta. (the term direct break-up stands for the situation in which neither projectile fragment fuses with the target) (iii) ICF following the breakup, when one of the fragments fuses with the target. (iv) $\mathrm{CF}$ following the break-up, when all projectile fragments fuse with the target. (v) CF as a single step mechanism, not produced by break-up. The first process $(i)$ should not affect the complete fusion $(v)$, since they are concerned with different partial waves. The other three break-up processes (ii, iii and $i v$ ) may influence the fusion (v) cross section. When one measures the direct break-up cross section, actually one is measuring the sum of the $(i)$ and (ii) processes. When one measures the complete fusion cross sections of systems with weakly bound nuclei, actually one is measuring the sum of the cross sections of two processes: $i v$ and $v$. When one measures the sum of CF and ICF cross sections, three mechanisms ( $i i i, i v$ and $v$ ) are mixed together and therefore the possible fusion suppression that can be observed is due to the effect of the direct break-up corresponding to low angular momenta (ii).

Recent theoretical results[13, 14] suggest that the differences in the direct break-up cross sections are not reflected on the values of the fusion cross sections, in agreement with experiments[15, 16, 17] that measure the direct break-up cross sections for ${ }^{6,7} \mathrm{Li}$ and ${ }^{6} \mathrm{He}$ on ${ }^{208} \mathrm{~Pb}$ and ${ }^{209} \mathrm{Bi}$. The results show that although the break-up process for ${ }^{6} \mathrm{Li}$ has cross sections that are one or two orders of magnitude larger than for ${ }^{7} \mathrm{Li}$, the total fusion $(\mathrm{CF}+\mathrm{ICF})$ cross sections for the two $\mathrm{Li}$ isotopes are similar.

Another approach to the study of the influence of the break-up on the fusion cross section is through the analysis of the behavior of the energy dependence of the real and imaginary parts of the optical potentials at near barrier energies. The presence or absence of the well known threshold anomaly is an indication of the coupling strengths of the elastic channel with the inelastic scattering or other direct reaction channels and it is directly related with the fusion cross section behavior at near barrier energies.

In this paper we discuss experimental results on the fusion and/or elastic and inelastic scattering of the ${ }^{6,7} \mathrm{Li},{ }^{9} \mathrm{Be}$ $+{ }^{27} \mathrm{Al},{ }^{64} \mathrm{Zn},{ }^{138} \mathrm{Ba}$ systems. At the end of the paper we try to give a very simple picture of the relation between the break-up and fusion processes.

The fusion experiments were performed at the 8UD Pelletron Laboratory of the Univeristy of São Paulo and at the 20UD Tandar Laboratory of the CNEA, Buenos Aires. The energy ranges were from the Coulomb barriers to twice or three times these values. Different experimental methods were used. The complete fusion cross sections of ${ }^{9} \mathrm{Be}+$ ${ }^{64} \mathrm{Zn}[4,5]$ were measured by the in-beam and off-beam gamma ray spectroscopy methods[18] at São Paulo, using two germanium detectors with Compton suppression, for four beam energies. Individual evaporation channels were observed for the CF, and no ICF was observed. The total fusion of ${ }^{6,7} \mathrm{Li}+{ }^{27} \mathrm{Al},{ }^{64} \mathrm{Zn}$ [11] were measured at Tandar, using a time of flight detection system especially developed for these measurements. One microchannel plate and one surface barrier silicon detector PIPS were used as start and stop detectors, respectively, with a $1.7 \mathrm{~m}$ flight distance. Seven beam energies were measured, and angular distributions were obtained for five angles, from $10^{\circ}$ to $30^{\circ}$, at three energies, for each system. The timing resolution was good enough to separate residual nuclei with masses differing by one unit of mass. The total fusion of ${ }^{9} \mathrm{Be}+{ }^{27} \mathrm{Al}$ [8] was measured, for ten beam energies, at Tandar, with a large ionization chamber followed by one surface barrier detector. Angular distributions were obtained for four angles, from $10^{0}$ to $20^{\circ}$, at four energies. The $\mathrm{E}-\Delta \mathrm{E}$ telescope had resolution to separate residual nuclei differing by one unit of atomic number. The scattering experiments were performed at São Paulo[2, 5, 7, 8], with an array of nine silicon surface barrier detectors, with energy resolution good enough to resolve elastic and inelastic peaks. Measurements at backward angles up to $165^{\circ}$ were performed.

The experiments on elastic and inelastic scattering of ${ }^{6,7} \mathrm{Li}+{ }^{138} \mathrm{Ba}[2,4,7]$ and ${ }^{9} \mathrm{Be}+{ }^{64} \mathrm{Zn} \mathrm{[4,} \mathrm{5,} \mathrm{7]} \mathrm{systems}$ have shown that the usual threshold anomaly of the optical potential is present in the ${ }^{7} \mathrm{Li}$, but not in the ${ }^{6} \mathrm{Li}$ and ${ }^{9} \mathrm{Be}$ scattering. This fact is in agreement with the results for the ${ }^{6,7} \mathrm{Li}$ $+{ }^{208} \mathrm{~Pb}$ system[19] and it is interpreted[2, 4, 5, 7, 19, 20] as the effect of the strong coupling of the elastic channel with the first ${ }^{7} \mathrm{Li}$ excited state channel. So, when the energy of the first excited state is lower than the dissociation energy, as for the ${ }^{7} \mathrm{Li}$ nucleus, the inelastic coupling predominates over the break-up process and the threshold anomaly is present. On the other hand, when the opposite situation occurs, as for ${ }^{6} \mathrm{Li}$ and ${ }^{9} \mathrm{Be}$ nuclei, the break-up process predominates over the inelastic coupling and the threshold anomaly vanishes. Therefore, the break-up of ${ }^{6} \mathrm{Li}$ and ${ }^{9} \mathrm{Be}$ inhibit their inelastic excitation and, consequently, does not allow the occurrence of the usual fusion cross section enhancement, relative to the predictions of one dimensional barrier penetration models. From this interpretation, at near barrier energies, the break-up and reaction cross sections for the ${ }^{6} \mathrm{Li}$ induced reactions should be higher than for the ${ }^{7} \mathrm{Li}$, but the fusion cross section should be smaller. Figure 1 shows the real and imaginary parts of the optical potential for the ${ }^{7} \mathrm{Li}+{ }^{138} \mathrm{Ba}$ system. The full dots (curves) are the values (fit) of the optical potential considering only the elastic scattering data. The open triangles (dashed curves) are the values (fit) when the coupling of the first excited state of the ${ }^{7} \mathrm{Li}$ is considered.

The influence of the break-up on the fusion cross section, in the following analysis, is investigated by the comparison of the fusion excitation functions of several medium-light systems, above barrier energies: the weakly bound ${ }^{6,7} \mathrm{Li}$ and ${ }^{9} \mathrm{Be}$, and the strongly bound ${ }^{16} \mathrm{O}$ and ${ }^{11} \mathrm{~B}$ nuclei as projectiles and the ${ }^{27} \mathrm{Al}$ and ${ }^{64} \mathrm{Zn}$ nuclei as targets $[1,4,5,8,11,21$ - 


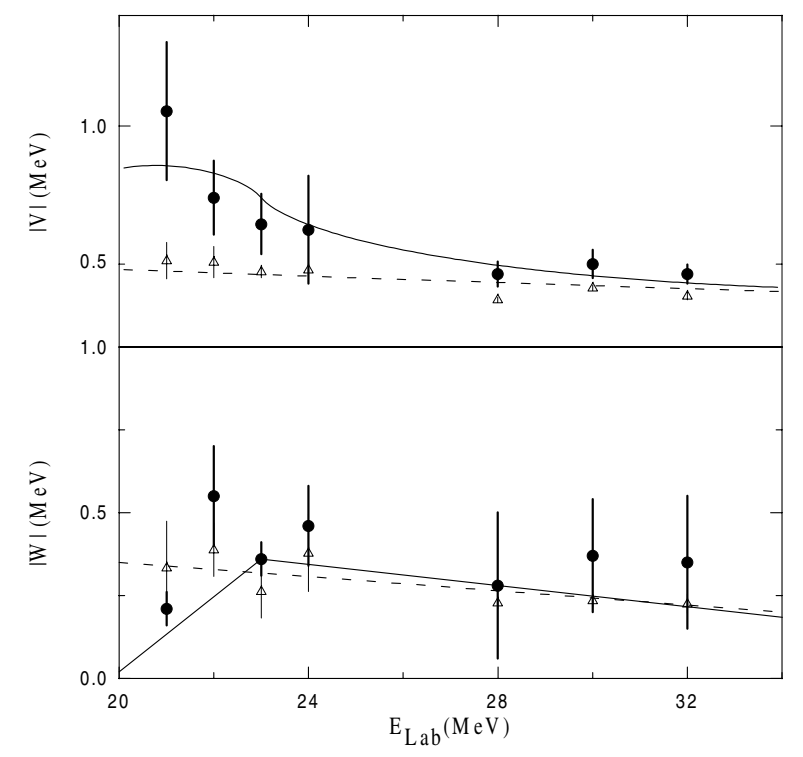

Figure 1. Values of the real and imaginary part of the optical potential at the radius of sensitivity equal to $11.27 \mathrm{fm}$ for the ${ }^{7} \mathrm{Li}+$

${ }^{138} \mathrm{Ba}$. The lines correspond to the dispersion relation calculations: full line and dots - optical model calculations, dashed line and open triangles - coupled channel calculations. The error bars correspond to the variation of one unity of the $\chi^{2}$ value.

23]. Each fusion excitation function was fitted by one dimensional barrier penetration models. If the direct break-up process had an important influence on the fusion cross section, the derived barrier parameters should have anomalous values. Reasonable fits were obtained for all the systems, and the barrier parameters agree with the values from the systematic study[24], within the usual fluctuations around the average values. The fusion excitation functions for these systems are very similar, regardless the presence or absence of weakly bound nuclei, as it is shown in Figs. 2 and 3. Furthermore, the comparison of the fusion excitation functions of systems of this mass region, leading to the same compound nuclei[8], do not show any dependence on the projectile separation energy. Also, the ratio fusion / reaction cross sections does not follow any systematic dependence with the separation energies[8], neither does the maximum measured experimental fusion cross sections [8]. Therefore, there are strong signatures that there is no total fusion cross section hindrance, above barrier energies, for these medium mass systems.

On the other hand, from the complete fusion cross sections of ${ }^{9} \mathrm{Be}+{ }^{208} \mathrm{~Pb}[3]$ and ${ }^{6,7} \mathrm{Li}+{ }^{209} \mathrm{Bi}[6,12]$ it is observed a strong fusion suppression at energies above the barrier. The conclusion from all the mentioned data on weakly bound stable nuclei is that the long range Coulomb break-up is responsible for the fusion suppression at energies above the barrier, and this process is predominant only for heavy targets.

One can make a conjecture that the direct break-up cross sections for the three weakly bound nuclei may be large and quite different for each of these projectiles, and they may increase the reaction cross sections, but they do not affect the total fusion cross section ( $\mathrm{CF}+\mathrm{ICF})$, at least within the experimental uncertainties. This effect has been recently sug

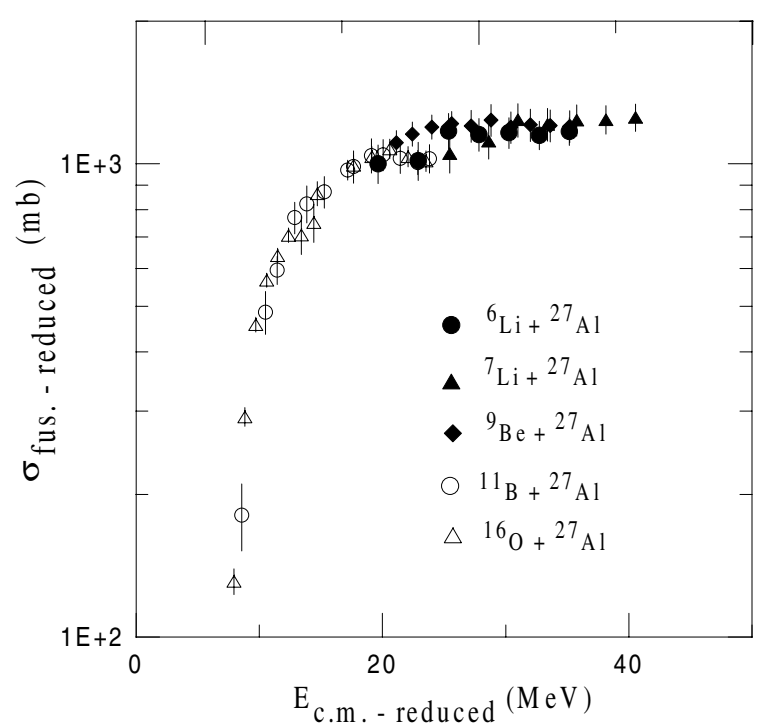

Figure 2. Fusion excitation functions for the ${ }^{6} \mathrm{Li},{ }^{7} \mathrm{Li},{ }^{9} \mathrm{Be},{ }^{11} \mathrm{~B}$, ${ }^{16} \mathrm{O}+{ }^{27} \mathrm{Al}$ systems. The values for the Coulomb barrier can be found in Ref.11. The reference system for reduced values was the ${ }^{6} \mathrm{Li}+{ }^{27} \mathrm{Al}$.

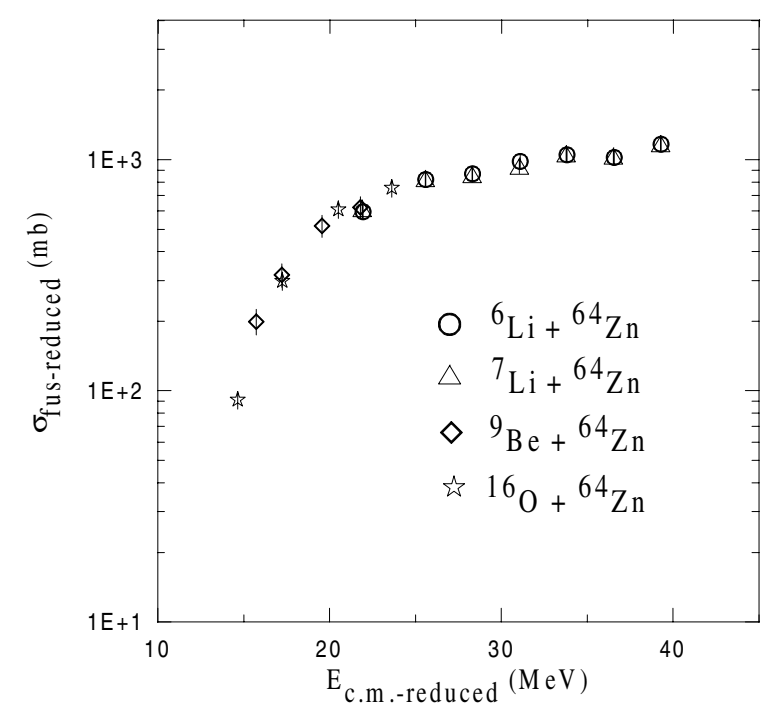

Figure 3. Fusion excitation functions for the ${ }^{6} \mathrm{Li},{ }^{7} \mathrm{Li},{ }^{9} \mathrm{Be},{ }^{16} \mathrm{O}+$ ${ }^{64} \mathrm{Zn}$ systems. The values for the Coulomb barrier can be found in Ref.11. The reference system for reduced values was the ${ }^{6} \mathrm{Li}+$ ${ }^{64} \mathrm{Zn}$.

gested by Keeley et al[13] for light systems, and verified by Kolata[25] for heavy systems. It is also in agreement with the conclusions drawn from low energy elastic scattering of ${ }^{6,7} \mathrm{Li}$ on heavy targets[2,19].

We conclude that, at above barrier regime, the direct break-up that does not lead to complete fusion or incomplete fusion is the Coulomb break-up that takes place at large distances or for trajectories corresponding to large angular momenta (process $(i)$ ). We conclude that it does not affect the fusion mechanism, although it affects the elastic scattering and reaction cross section. The break-up process that might inhibit or enhance the complete fusion occurs at small distances or central trajectories, where both Coulomb and nuclear break-ups are important. For heavy targets it has 
been observed $[3,6,12]$ that although the ICF cross sections were found to be significant, when compared to $\mathrm{CF}$ cross sections (of the order of $30 \%$ of $\mathrm{CF}$ above the barrier), at high energies the sum of the measured $\mathrm{CF}+\mathrm{ICF}$ cross sections agree with the predictions of one dimensional barrier penetration models that do not consider the break-up effect. So, for heavy targets it is found that the effect of the direct break-up, corresponding to low partial waves (process (ii)), on the total fusion is negligible. For the ${ }^{9} \mathrm{Be}+{ }^{64} \mathrm{Zn}$ system, where the $\mathrm{CF}$ and ICF cross sections could be measured separately $[4,5]$, the ICF cross section was found to be negligible. Therefore, we expect that the ICF is not so important for light systems, at energies above the barrier, as it is for the heavy ones. Furthermore, if the ICF is not important, the CF following break-up (process (iv)) is not expected to be relevant. Therefore, the present results for the ${ }^{6,7} \mathrm{Li},{ }^{9} \mathrm{Be}$ $+{ }^{27} \mathrm{Al},{ }^{64} \mathrm{Zn}$ systems show that the total fusion cross section $(\mathrm{CF}+\mathrm{ICF})$ is also not inhibited for medium-light systems, due to break-up effects. Also, although the behavior of ${ }^{6} \mathrm{Li}$ and ${ }^{9} \mathrm{Be}$ scattering are qualitatively different from ${ }^{7} \mathrm{Li}$ scattering $[2,4,5,7,19]$, this is not reflected in the total fusion cross sections.

For a complete understanding of the influence of the break-up process on the fusion cross section, complete fusion, incomplete fusion, direct break-up, and elastic scattering have to be measured with enough degree of precision to evaluate the influence of each process separately. While such complex experiments are lacking the relevant information has to be extracted from the available data and in addition further theoretical models and calculations deserve to be developed. More data at sub-barrier energies are also required.

\section{Acknowledgments}

The authors wish to thank the Fundação Vitae and Fundación Antorchas for their financial support that allowed the collaboration Brazil-Argentina. The Brazilian authors also would like to thank the CNPq for their partial financial support. Some of us (A.J.P., J.O.F.N. and J.E.T) are fellows of the Carrera del Investigador Científico of the CONICET, and M.R.S. is a postdoctoral fellow of the Carrera del Investigador Científico of the CONICET.

\section{References}

[1] R.M. Anjos, R.M. Anjos, C. Muri, S.B.Moraes, R. Cabezas, P.R.S. Gomes, A.M.M. Maciel, G.M. Santos, C. Tenreiro, and R. Liguori Neto, J. Phys. G 23, 1423 (1997).

[2] A.M. Maciel, P.R.S. Gomes, J. Lubian, R.M. Anjos, R. Cabezas, S.B. Moraes, C. Muri, M.M. Sant'Anna, G.M. Santos, R. Liguori Neto, N. Added, N. Carlin, and C. Tenreiro, Phys. Rev. C 59, 2103 (1999).

[3] M. Dasgupta, D.J. Hinde, R.D. Butt, R.M. Anjos, A.C. Berriman, N. Carlin, P.R.S. Gomes, C.R. Morton, A.S. Toledo, and K. Hagino, Phys. Rev. Lett. 82, 1395 (1999).

[4] P.R.S. Gomes, R.M. Anjos, J. Lubian, A.M.M. Maciel, S.B. Moraes, C. Muri, M.M. Sant'Anna, J.J.S. Santos, I. Padrón,
R. Cabezas, R. Liguori Neto, and N. Added, Heavy Ion Phys. 11, 361 (2000).

[5] S. B. Moraes, P.R.S. Gomes, J. Lubian, J.J.S. Alves, R.M. Anjos, M.M. Sant'Anna, I. Padron, C. Muri, R. Liguori Neto, and N. Added, Phys. Rev. C 61, 064608 (2000).

[6] M. Dasgupta, S.B. Moraes, P.R.S.Gomes, D. J. Hinde, R.D. Butt, R.M. Anjos, A. C. Berriman, N. Carlin, C. R. Morton, and A. S. Toledo, Int. Workshop on Fusion Dyn. at the Extremes, Dubna, Russia (2000), pag. 254.

[7] J. Lubian, I. Padrón, A.M.M. Maciel, P.R.S. Gomes, R. M. Anjos, R. Liguori Neto, and N. Added, Phys. Rev. C 64, 027601 (2001).

[8] R.M. Anjos, C. Muri, J. Lubian, P.R.S. Gomes, I. Padrón, J. J.S. Alves, G.V. Marti, J.O. Fernández Niello, A.J. Pacheco, O. A. Capurro, D. Abriola, J.E. Testoni, M. Ramirez, R. Liguori Neto, and N. Added, Phys. Lett. B 534, 45 (2002).

[9] A. Mukherjee, M. Dasgupta, D.J. Hinde, H. Timmers, R.D. Butt, and P.R.S. Gomes, Phys. Lett. B 526, 295 (2002).

[10] W.H.Z. Cardenas,L.F. Canto, R. Donangelo, M.S. Hussein, J. Lubian, and A. Romanelli, Nucl. Phys. A703, 633 (2002).

[11] I. Padron, P.R.S. Gomes, R.M. Anjos, J. Lubian, C. Muri, and J.J.S. Alves, G.V. Marti, M. Ramírez, A.J. Pacheco, O.A. Capurro, J.O. Fernández Niello, J.E. Testoni, D. Abriola, and M.R. Spinella, Phys. Rev. C 66, 044608 (2002).

[12] M. Dasgupta et al., in press in Phys. Rev. C (Rapid communication).

[13] N. Keeley, K.W. Kemper, and K. Rusek, Phys. Rev. C 65, 014601 (2001).

[14] A Diaz-Torrez, I.J. Thompson, and W. Scheid, Nucl. Phys. A703, 83 (2002).

[15] E.F. Aguillera, J.J. Kolata, F.D. Becchetti, P.A. de Young, J.D. Hinnefeld, A. Horvath, L.O. Lamm, Hye-Young Lee, D. Lizcano, E. Martinez, Quiroz, P. Mohr, T.W. O’Donnell, D.A. Roberts, and G. Rogachev, Phys. Rev. C 63, 061603 (2001).

[16] G.R. Kelly, N.J. Davis, R.P. Ward, B.R. Fulton, G. Tungate, N. Keekey, K. Rusek, E.E. Bartosz, P.D. Cathers, D.D. Caussyn, T.L. Drummer, and K.W. Kemper, Phys. Rev. C 63, 024601 (2001).

[17] C. Signorini, M. Mazzocco, G.F. Prete, F. Soramel, L. Stroe, A. Andrigheto, I.J. Thompson, A. Vitturi, A. Brondi, M. Cinausero, D. Fabris, E. Fioretto, N. Gelli, J.Y. Guo, G. La Rana, Z.H. Liu, F. Lucarelli, R. Moro, G. Nebbia, M. Trotta, E. Vardaci, and G. Viesti, Europ. Phys. J. A10, 249 (2001).

[18] P.R.S. Gomes, T.J.P. Penna, R. Liguori Neto, J.C. Acquadro, C. Tenreiro, E. Crema, N. Carlin Filho, and M. M. Coimbra, Nucl. Instr. and Methods in Phys. Research A280, 395 (1989).

[19] N. Keeley, S.J. Bennet, M.N. Clarke, B.R. Fulton, G. Tungate, P.V. Drumm, M.A. Nagarajan, and J.L. Lilley, Nucl. Phys. A 571, 326 (1994); N. Keeley, K. Rusek, Phys. Lett. B 427, 1 (1998).

[20] Y. Sakuragi, M. Yahiro, and M. Kamimura, Prog. Theor. Phys. 70, 1047 (1983).

[21] R.M. Anjos, V. Guimarães, E.M. Szanto, N. Carlin Filho, M.M. Coimbra, L. Fante Jr., M.C.S. Figueira, C.F. Tenreiro, and A. Szanto Toledo, Phys. Rev. C 42, 354 (1990). 
[22] C. Tenreiro, J.C. Acquadro, P.A.B. Freitas, R. Liguori Neto, G. Ramirez, N. Cuevas, P.R.S. Gomes, and J. Copnell, Proc. Workshop on Heavy Ion Fusion: Exploring the Variety of Nuclear Properties; Padova, Italia (1994), 98.

[23] B.B. Back, R.R. Betts, C. Gaarde, J.S. Larsen, E. Michelsen, and Tai-Kuang-Hsi, Nucl. Phys. A285, 317 (1977); R.L. Kozub, N. A. Lu, I.M. Miller, and D.Logan, Phys. Rev. C
11, 1497 (1976); J. Einzen, I. Tserruya, Y. Eyal, Z. Fraenkel, and M.H. Hilmman, Nucl. Phys. A291, 459 (1977).

[24] L.C. Vaz, J.M. Alexander, and G.R. Satchler, Phys. Rep. 69, 373 (1981).

[25] J.J. Kolata, Phys. Rev. C 63, 061604R (2001). 